\title{
Call for Papers: Applications of Next Generation CRISPR-derived Technologies
}

\author{
Due date for submissions: August 1, 2020
}

CRISPR-based genome editing has revolutionized experimental biology and is being harnessed for human gene therapy. The fundamental capability of guiding a nucleotide-altering enzyme to a specific genomic or RNA sequence by means of a short RNA guide (sgRNA) allows for precise nucleic acid alterations to be made in human cells and a wide variety of different organisms. While initial applications of the CRISPR-Cas system were primarily based on the Cas9 endonuclease function, protein engineering has enabled a variety of other DNA- and RNA-altering functions to be guided by the Cas-sgRNA mechanism. Human Gene Therapy is now seeking original papers and review articles that describe the design, optimization, and application of these next-generation CRISPR approaches, including, but not limited to, the following technologies:

- Base editing

- Epigenome editing

- CRISPR-transactivation

- RNA editing

- Prime editing

- Anti-CRISPR proteins

- Novel Cas-based fusion proteins that confer novel functions, sequence-targeted by Cas-sgRNA

Demonstrations of the utility of one or more of such technologies in animal models of specific human diseases, including monogenic disorders, is of particular interest.

For questions regarding submissions or for technical assistance, contact HGT's Managing Editor: Mary Mazzanti at Mary.Mazzanti@umassmed.edu

Please visit our website for Instructions for Authors at www.liebertpub.com/HGT to assist with manuscript preparation.

\section{Submit all manuscripts for consideration via our peer review submission site: https://mc.manuscriptcentral.com/hum}

